\title{
Role of capsule endoscopy in suspected or established Crohn's disease in real practice
}

\author{
Hyun Joo Jang \\ Department of Internal Medicine, Hallym University School of Medicine, Dongtan Sacred Heart Hospital, Hwaseong, Korea
}

\section{Article: Practice patterns and clinical significance of use of capsule endoscopy in suspected and established Crohn's disease (Intest Res 2017;15:467-474)}

Capsule endoscopy (CE) and balloon-assisted enteroscopy are advanced diagnostic and therapeutic modalities used for the management of suspected small bowel disease. CE is a noninvasive and sensitive option to evaluate the small intestine in patients presenting obscure gastrointestinal bleeding, CD, polyposis syndromes, celiac disease, or other suspected small intestinal diseases. ${ }^{1} \mathrm{CD}$ is a chronic IBD that may affect any part of the gastrointestinal tract; the incidence of CD of the small intestine is approximately $40 \%$ to $60 \%$. CD is diagnosed based on a combination of endoscopic, radiological, histological and biochemical evaluation, as well as clinical symptoms.

A recent clinical guideline in Canada suggests that CE is recommended for patients presenting clinical symptoms compatible with CD, in the presence of a negative ileocolonoscopic examination and radiological studies. CE is also recommended in established CD patients showing clinical symptoms that are unexplained by an ileocolonoscopic examination or radiological studies. CE is recommended in established CD patients to assess small intestinal mucosal healing. CE is needed in patients in whom ileocolonoscopic examination and/or radiological studies might not successfully detect the recurrence of small bowel CD after a colectomy. This guideline in Canada suggests that CE is not

Received September 20, 2017. Revised September 20, 2017. Accepted September 20, 2017.

Correspondence to Hyun Joo Jang, Department of Internal Medicine, Hallym University School of Medicine, Dongtan Sacred Heart Hospital, 7 Keunjaebong-gil, Hwaseong 18450, Korea. Tel: +82-31-8086-2450,

Fax:+82-31-8086-2029, E-mail:jhj1229@hallym.or.kr

Financial support: None. Conflict of interest: None. recommended in patients with chronic abdominal pain or diarrhea without any evidence of elevated biomarkers. ${ }^{2}$

The European Society of Gastrointestinal Endoscopy (ESGE) guidelines recommend that CE is the preferred initial diagnostic option for the evaluation of the small intestine in patients presenting suspected CD and demonstrating negative ileocolonoscopic examination in the absence of obstructive symptoms or radiological stenosis. ESGE also recommends that careful patient selection based on careful assessment of clinical symptoms and serological or fecal inflammatory biomarkers is an important factor to improve the diagnostic accuracy and yield of CE in in patients presenting with suspected CD. ESGE recommends the use of radiological imaging such as CT enterography or MR enterography, to investigate the extent of $\mathrm{CD}$ and/or extraluminal involvement in patients with a known diagnosis of $\mathrm{CD}{ }^{3}$

The current issue of Intestinal Research has published a report by Kim et al. ${ }^{4}$ describing a retrospective study focusing on the current practice pattern for CE in patients with suspected CD or those with established CD. In this study, the overall diagnostic yield of a CE was 59.7\% (34/57) in patients with suspected CD, and $28.1 \%$ patients $(16 / 57)$ were diagnosed with CD based on CE findings. A recent metaanalysis showed that the diagnostic yield of CE in patients with suspected CD was significantly higher than that of a small bowel follow through (SBFT), enteroclysis and CT enterography (CE $52 \%$ vs. SBFT/small bowel enteroclysis $16 \%$ and incremental yield $32 \% ; P<0.001 ; 95 \% \mathrm{CI}, 16 \%-48 \%$ : CE $68 \%$ vs. CT enterography $21 \%$ and incremental yield $47 \% ; P<0.001 ; 95 \%$ CI, 31\%-63\%). ${ }^{5}$ Tukey et al. ${ }^{6}$ reported that careful patient selection based on the International Confer-

\footnotetext{
๑ Copyright 2017. Korean Association for the Study of Intestinal Diseases. All rights reserved.

This is an Open Access article distributed under the terms of the Creative Commons Attribution Non-Commercial License (http://creativecommons.org/licenses/by-nc/4.0)

which permits unrestricted non-commercial use, distribution, and reproduction in any medium, provided the original work is properly cited.
} 
ence on Capsule Endoscopy (ICCE) criteria is important to increase the positive predictive value of CE. In this study, CD was defined based on the similar criteria with ICCE criteria, which included the presence of at least 2 of the following: abdominal pain or diarrhea, iron deficiency anemia, elevated ESR or CRP, hypoalbuminemia, extraintestinal manifestations, and a family history of IBD. The positive predictive value of a CE varies based on the patient population and the CE criteria utilized to diagnose $\mathrm{CD}$. Careful patient selection is important to evaluate the small bowel using CE in patients with suspected CD.

In this study, the most common indication to perform a CE was to obtain an initial diagnosis in patients with suspected CD (54.4\%) and to investigate the extent and disease activity in patients with established CD. There was no instance of capsule retention observed in patients with suspected $\mathrm{CD}$ and only 1 instance of capsule retention in patients with established CD $(7.1 \%, 1 / 14)$. Based on radiological imaging studies, it has been observed that the risk of capsule retention in patients with suspected CD is quite low (approximately $1.6 \%$ ) in the absence of obstructive symptoms or known stenosis. ${ }^{7}$ However, the risk of capsule retention is high (approximately 13\% in patients with established CD). ${ }^{7}$ Recent guidelines suggest use of a patency capsule prior to performing a CE to decrease the risk of capsule retention in patients with known or suspected stenosis of the small intestine. ${ }^{2}$ Guidelines of the Korean Gut Image Study Group of the Korean Society of Gastrointestinal Endoscopy recommend obtaining cross-sectional radiological imaging or using a patency capsule prior to performing a CE in patients with established $\mathrm{CD}^{8}{ }^{8}$

A retrospective study has reported that capsule retention rates were similar between patients undergoing $\mathrm{CE}$ without a patency capsule and those undergoing CE after a negative patency capsule study ( $2.3 \%$ vs. $2.1 \%, P=0.9) .{ }^{9}$ However, it was observed that performing a CE after a positive result of a patency capsule test was associated with a high risk of capsule retention $(11.1 \%, P=0.01) .{ }^{9}$ Selective use of a patency capsule in patients with risk factors of capsule retention showed a similar retention rate and reduced costs of the patency capsule compared to that of nonselective use of patency capsule in patients with established $\mathrm{CD}{ }^{9}$ A patency capsule is currently not available for use in Korea but is expected to become commercially available soon. Therefore, capsule retention in patients with established CD is common and cross-sectional radiological imaging or use of a patency capsule prior to performing a CE is recommended in patients with established CD to reduce capsule retention rates.

Endoscopic investigation of the small intestine is useful to assess mucosal healing and/or early recurrence of inflammation. ${ }^{10} \mathrm{CE}$ may affect therapeutic plans in patients with established or suspected CD. This study reported that therapeutic plans had been modified in $70.2 \%$ of patients with suspected CD and $57.1 \%$ of patients with established CD had current medications kept or specific medications added. Overall therapeutic impact of CE was observed in $70.2 \%$ of patients with suspected CD and $50.0 \%$ of patients with established CD despite the small number of established CD patients studied. Mucosal healing is an important treatment goal in patients with CD. However, the clinical response to treatment may not correlate well with mucosal healing in patients with CD. One study reported that high CRP, fecal calprotectin, or a combination of both was poorly correlated with significant inflammatory CE findings in the small intestine in established CD patients. CE affected therapeutic changes in $>50 \%$ of established CD patients.

A limitation of this study is that because this was a retrospective study and only a small number of patients with established CD were evaluated, the overall diagnostic and therapeutic yields might have included a selection bias. Therefore, large-scale prospective studies are needed to determine the role of CE in patients diagnosed with CD. Regardless of this limitation, this study provides useful information about the practical use of CE in patients with suspected and established CD.

\section{REFERENCES}

1. Pandey V, Ingle M, Pandav N, et al. The role of capsule endoscopy in etiological diagnosis and management of obscure gastrointestinal bleeding. Intest Res 2016;14:69-74.

2. Enns RA, Hookey L, Armstrong D, et al. Clinical practice guidelines for the use of video capsule endoscopy. Gastroenterology 2017;152:497-514.

3. Pennazio M, Spada C, Eliakim R, et al. Small-bowel capsule endoscopy and device-assisted enteroscopy for diagnosis and treatment of small-bowel disorders: European Society of Gastrointestinal Endoscopy (ESGE) clinical guideline. Endoscopy 2015;47:352-376.

4. Kim Y, Jeon SR, Choi SM, et al. Practice patterns and clinical significance of use of capsule endoscopy in suspected and established Crohn's disease. Intest Res 2017;15:467-474. 
5. Dionisio PM, Gurudu SR, Leighton JA, et al. Capsule endoscopy has a significantly higher diagnostic yield in patients with suspected and established small-bowel Crohn's disease: a metaanalysis. Am J Gastroenterol 2010;105:1240-1248.

6. Tukey M, Pleskow D, Legnani P, Cheifetz AS, Moss AC. The utility of capsule endoscopy in patients with suspected Crohn's disease. Am J Gastroenterol 2009;104:2734-2739.

7. Cave D, Legnani P, de Franchis R, Lewis BS; ICCE. ICCE consensus for capsule retention. Endoscopy 2005;37:1065-1067.
8. Song HJ, Shim KN. Current status and future perspectives of capsule endoscopy. Intest Res 2016;14:21-29.

9. Nemeth A, Kopylov U, Koulaouzidis A, et al. Use of patency capsule in patients with established Crohn's disease. Endoscopy 2016;48:373-379.

10. Kim M, Jang HJ. The role of small bowel endoscopy in small bowel Crohn's disease: when and how? Intest Res 2016;14:211217. 